\title{
The Role of Endoscopy in the Diagnosis of Digestive Pathology
}

\author{
Iuliu SCURTU ${ }^{\text {ac }}$, Gavril GIURGIU ${ }^{\text {ac }}$, Cosmin PESTEAN ${ }^{\text {ad }}$, Flaviu TABARAN ${ }^{\text {ae }}$, Mircea MIRCEAN ${ }^{\text {abc }}$ \\ ${ }^{a}$ University of Agricultural Science and Veterinary Medicine, Manastur Street 3-5, 400372, Cluj Napoca \\ 'Internal Medicine Department \\ ${ }^{d}$ Anesthesiology Department \\ ${ }^{e}$ Morphopathology and Necropsy Diagnosis \\ *Corresponding author: mircea.mircean@usamvcluj.ro
}

Bulletin UASVM Veterinary Medicine 72(2) / 2015,

Print ISSN 1843-5270; Electronic ISSN 1843-5378

DOI:10.15835/buasvmcn-vm: 11643

\begin{abstract}
Nowadays, endoscopy represents one of the most useful tools in the diagnosis of digestive pathologies in human beings and animals. Whether we are talking about upper or lower gastrointestinal tract endoscopies, these procedures dramatically increase the capacity to diagnose digestive disorders in dogs and cats. The aim of the present study is to investigate the role of endoscopy in the diagnosis of digestive pathologies.

This paper represents our 5 years' experience in digestive endoscopy. During this period we performed more than 150 digestive procedures in dogs and we diagnosed a heteromorphic digestive pathology. Clinical cases were represented by client-owned dogs that were referred to our clinic with a suspicion of digestive pathology.. We diagnosed erosive esophagitis and esophageal foreign bodies, hemorrhagic gastritis and ulcers, gastric and intestinal tumors, infiltrative enteritis, cecal intussusception, and colorectal carcinoma. It must be stressed that these diagnoses were missed using other investigative methods, and we can sustain that endoscopy dramatically increases our diagnostic capacity. Moreover in some conditions (for e.g. esophageal or gastric foreign bodies) endoscopy could also be used as a therapeutic tool.

We recommend endoscopy in every case where there is a suspicion of digestive pathology and where other diagnostic tools did not reveal any abnormalities. The main disadvantages of endoscopy were the need for anesthesia in case of upper G.I. series and the cost of the procedure. We did not encounter any complications related to anesthesia or the procedure itself.
\end{abstract}

Keywords: colonoscopy, dog, endoscopy

\section{INTRODUCTION}

Endoscopy has had major technological advancements in the past decades, causing great impact on diagnostic accuracy (Assirati, 2014). In human medicine, a fully flexible and maneuverable endoscope was developed and introduced in practice in the late 1960s (Javali, 2015) and thence better characterizing digestive lesions. Surgical procedures were better orientated knowing the location, type and aspect of the pathology. Compared to radiology and ultrasonography, endoscopy has a greater advantage in that it gives the physician a possibility to analyze tissue and moreover take a biopsy sample from the organ under investigation. It is a fundamental method of investigation of the digestive tract, and is important in the diagnosis and prognosis of a variety of gastrointestinal disorders (Lecoindre, 1999). Endoscopy as a noninvasive modality allows visual examination of the digestive mucosa and lumen and facilitates the procurement of specimens for biopsy, cytology, and culture (Tams, 1999). 
This paper represents our five years' experience in digestive endoscopy. We will list the type of digestive pathology we diagnosed, advantages, disadvantages and also limitations of endoscopy. We also want to see if endoscopy is more advantageous than ultrasonography and radiology in the diagnosis of digestive pathology.

\section{MATERIALS AND METHODS}

This study was carried out at the Internal Medicine Department of the Faculty of Veterinary Medicine of Cluj Napoca. It consisted of 150 dogs having digestive pathologies, with 67 being males, and 83 being females. The average age was 9.3 years. The chief complaints of the patients enrolled in the study were vomiting, diarrhea and tenesmus. Less frequently, we also noticed depression, decreased appetite or anorexia, and painful abdomen. Two types of flexible endoscopes (Pentax, tube length $130 \mathrm{~cm}$, tube diameter $13.2 \mathrm{~mm}$, and Karl Storz, tube length $300 \mathrm{~cm}$, tube diameter $9.2 \mathrm{~mm}$ ) were used. Prior to the beginning of the procedure, all the patients had a complete blood workup and a cardiac examination. For upper G.I. endoscopy, all the dogs were fasted for 12 hours before the procedure. Water was allowed. In the case of colonoscopy, the patients were fasted for 24 hours. As lavage solution, Fortrans ${ }^{\circledR}$ (1 paunch in 1 $\mathrm{L}$ of water) was used. The animals were given the solution at a dose of 25 to $30 \mathrm{~mL} / \mathrm{Kg}$. A minimum of two doses were administered at least two hours apart (Tams, 1999). In five patients, it was necessary to perform enema.

Patients were anesthetized for upper G.I. endoscopy. Butorphanol was used at a dose of 0.2 $\mathrm{mg} / \mathrm{Kg}$ and Propofol was administered I.V., slowly to effect. Usually, for colonoscopy, anesthesia was not needed. Sometimes, a light sedation was provided by using Diazepam and Butorphanol, which accounted for 5 cases only. 109 upper G.I. endoscopies and 41 colonoscopies were carried out.

\section{RESULTS AND DISCUSSIONS}

In our study, upper G.I. endoscopy (UGI) outnumbered colonoscopy. UGI was performed in $72.66 \%$ of cases (109 dogs) while colonoscopy was used in $27.34 \%$ of the cases ( 41 dogs). Males and females were equally represented.
Esophageal pathology represented $14.67 \%$ (16 cases) and gastroscopy was performed in 93 cases $(85.33 \%)$.

Esophageal pathology was less characteristic from the clinical point of view, so, endoscopy represented the best paraclinical investigation tool to image the esophageal mucosa. The most frequent type of pathology we identified was reflux esophagitis (Fig. 1C), in patients with chronic vomiting. Esophageal foreign bodies were diagnosed in five patients (Fig. 1A, 1B). In three out of five cases, we succeeded in relieving the obstruction. However, in two situations this was not feasible, and the patients were referred to the Surgery Department. Esophageal foreign bodies, mainly those with sharp edges, could induce petechiae and ulcers at the mucosal level, resulting in scar retraction or stenosis (Lecoindre and Cadore, 1993). We also identified a neostructure within the esophagus (Fig. 1D). The risk for complications and perforation after endoscopic foreign bodies removal increases when the foreign body has been in place for more than 3 days or the dog weighed less than $10 \mathrm{~kg}$ (Moon, 2012). Compare with contrast radiography, esophagoscopy is less definitive for diagnosing megaesophagus and other motility disorders, diverticulum, hiatal hernia, and compression, by periesophageal masses Besides the pathology we diagnosed, specific literature also talks about gastroesophageal intussusception (Shibly, 2014), esophageal fistula, megaesophagus, hiatal hernia and esophageal perforation (McCarthy, 2005).

Haemorrhagic gastritis and gastric ulcers were the most frequent pathologies diagnosed. 40 dogs out of 93 had haemorrhagic gastritis and gastric ulcers. 21 dogs out of 40 presented a recent history of NSAIDs or glucocorticoids administration. The lesions had different dimensions and aspects, being very small, ranging from $1 \mathrm{~mm}$ to $1-3 \mathrm{~cm}$ in diameter. Small lesions were localized mainly in the superficial layers of the gastric wall (mucosa), while larger lesions affected the deeper layers.

Gastric biopsies revealed hypertrophic gastritis (7 cases) and atrophic gastritis (8 cases). Gastric foreign bodies were diagnosed in 3 dogs. Normally, a gastric foreign body induces an acute or peracute pathology. Gastric foreign body removal is an emergency. In one patient with gastric foreign body, there was a history of vomiting episodes for 6 months, when the dog 

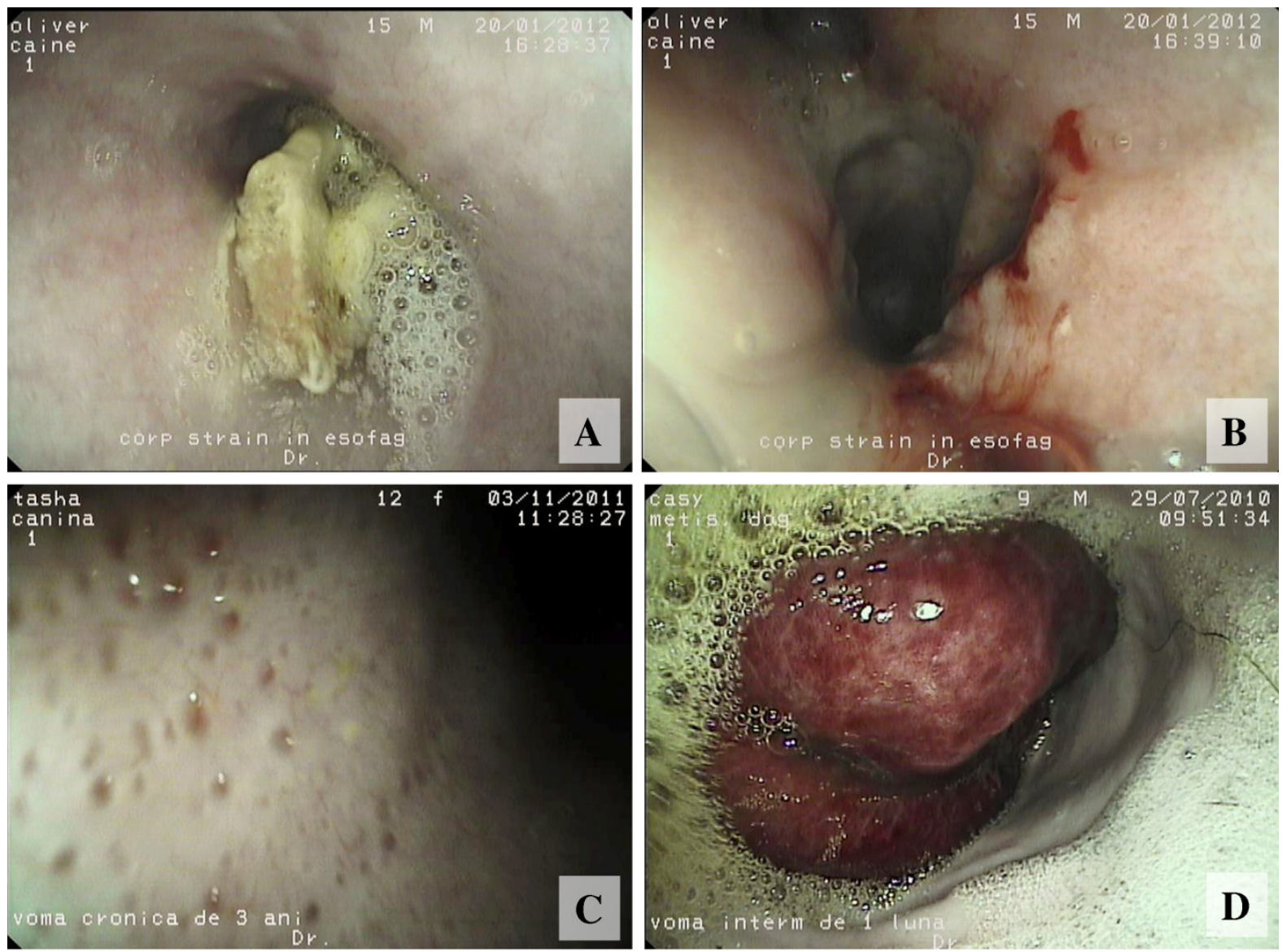

Fig. 1. A. Foreign body within esophagus. B. Esophageal mucosa's aspect after foreign body removal. C. Esophageal ulcers in a dog with chronic vomiting. D. Neostructure within esophagus.

was given some leftover food. The success rate of extracting gastric foreign bodies was 100\% (Fig. 2B). Gastric polyps were also diagnosed. They had small dimensions, and were considered to have a minor role in pathology, being a nonspecific finding. Gastric adenocarcinoma localized at the antrum level, was found in five dogs with an aspect quite characteristic for a neoplasia (Fig. 2A).

The chief complaint in patients with gastric pathology was vomiting, with a high frequency in dogs with hemorrhagic gastritis and gastric ulcers (5-6 times/day), and less often in patients suffering from atrophic or hypertrophic gastritis. According to the history, in dogs where we diagnosed gastric neoplasia, the onset of vomiting was at least 12 months prior to the diagnosis. In one case, vomiting onset was 36 months before presentation. Vomiting frequency increased as the cancer evolved. Patients with gastric foreign bodies also presented vomiting, but generalized abdominal pain was also noticed. In one case, in which the foreign body remained into the stomach for 6 months, the owner described a painful abdomen, without being able to identify clearly the painful area. After its extraction, the clinical signs improved.

All the patients with gastric pathologies were also scanned. A definitive diagnosis through ultrasonography was unrealistic. It brought little information regarding gastric pathologies. It was possible to characterize the thickness of the gastric wall through ultrasonography, but, it was impossible to describe the lesions localized at the surface of the gastric mucosa. Nevertheless, concerning gastric tumor, we succeeded in identifying an abnormal structure in the pylorus region. However, it was very difficult to characterize that mass. The main advantage of endoscopy in comparison to other paraclinical screening tools is biopsy. Biopsy sample characterization gives a physician all the information needed in order to put an accurate diagnosis.

We did not encounter any complications after performing upper G.I. endoscopies. The most 

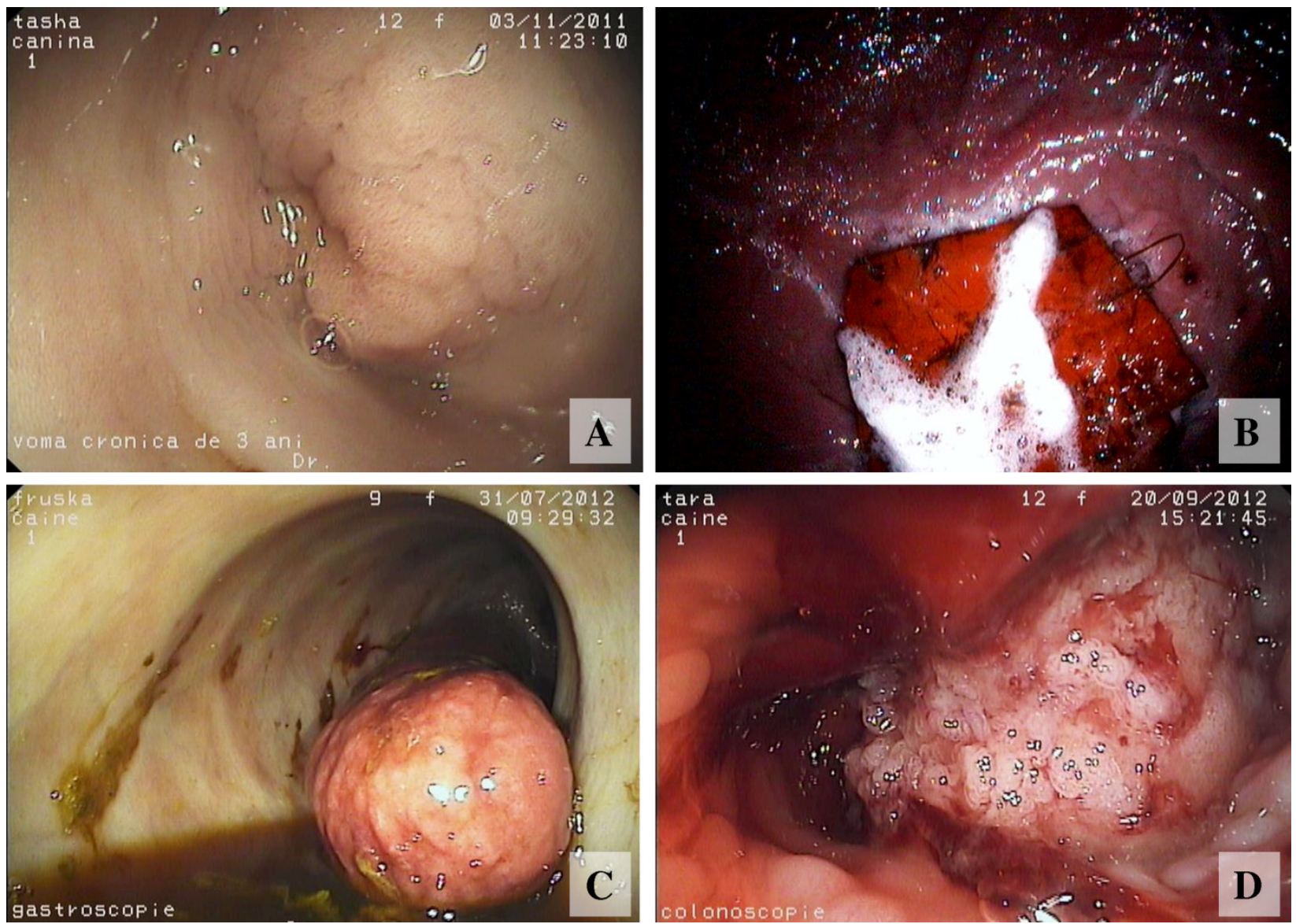

Fig. 2. A. Gastric adenocarcinoma. B. Gastric foreign body. C. Cecal intussusception. D. Colorectal carcinoma.

common complications of gastric endoscopy are: gastric and esophageal perforation, laceration of major blood vessels, decreased venous return due to gastric overdistension, acute bradycardia, gastric dilatation and volvulus, and mucosal hemorrhage (Veterinary endoscopy for small animal practitioner). In our case, the main problem faced was, approaching the pylorus when the stomach was distended with air. To solve this issue, the recommendation was air evacuation from the distended stomach (Tams, 1999).

Colonoscopy was performed in 41 dogs, where the main complaint was chronic diarrhea and/or diffuse abdominal pain. Chronic colitis was present in 8 cases, based on the biopsy sample. We also found ceco-colic intussusception (Fig. 2C), and colorectal carcinoma (Fig. 2D) was diagnosed in one patient too. The dog with the ceco-colic intussusception presented a severe painful abdomen and signs of shock. In the case of colorectal carcinoma, hematochezia was the primary problem. In 31 dogs, colonoscopy did not give any useful information to explain the clinical signs.

Ultrasonography and radiology were less useful than endoscopy in colorectal pathology. The thickness of the intestinal wall was the only abnormality found while performing ultrasonography. Ceco-colic intussusception was missed at ultrasonography. Large bowel radiology was even less characteristic when using ultrasonography; deeper characterization of intestinal wall (thickness, smooth or rough surface) could not be achieved.

Indications for performing colonoscopy include chronic large boweldiarrhea, chronic mixed bowel diarrhea and tenesmus, hematochezia, and excess fecal mucus associated with a formed stool (Leib, 2005). We did not stumble upon any complications while performing colonoscopy. A few complications are mentioned though: perforation of the colon, excessive bleeding and post procedural diarrhea. These are usually selflimiting and rarely require intervention (McCarthy, 2005). 


\section{CONCLUSIONS}

Digestive endoscopy is a modern investigative tool which increases the diagnostic capacity of our clinic. We recommend it as a routine procedure, mainly in a situation associated with chronic vomiting, diarrhea and hematochezia. Endoscopy is curative in the case of digestive foreign bodies. We consider that definitive diagnosis of chronic gastric pathology, gastric tumor or chronic intestinal disorders would have been impossible without endoscopy. Diagnosis of gastric ulcers and hemorrhagic gastritis might be oriented based on clinical examination, but the characterization of lesions and lesions extent could be properly performed by endoscopy. Colorectal carcinoma needs also endoscopic examination for a definitive diagnosis. Care must be taken in geriatric patients and those with heart problems because anesthesia could induce life-threatening complications

\section{REFERENCES}

1. Assirati FS, Hashimoto CL, Dib RA, Fontes LHS, Rodriguez NT, (2014), High definition endoscopy and narrow band imaging in the diagnosis of gastroesophageal reflux disease, ABCD Arq Bras Circ Dig, 27(1):59-65

2. Javali S, Madan M, Harendrakumar ML, Mahesh MS, (2015), Role of endoscopy in evaluating upper gastrointestinal tract lesions in rural population, J Dig Endosc, 6:59-65

3. Lecoindre $P$, (1999), An atlas of gastrointestinal endoscopy in dogs and cats, Waltham Focus, 3:1-9

4. LecoindreP, Cadore JL, (1994) Les affections de l'oesophagedescarnivoresdomestiques. PratiqueMédicale et Chirurgicale de l'Animal de Compagnie, 2:25-43

5. Leib M, (2005), Endoscopic examination of the small and large intestines, Proceedings of the NAVC, Orlando, Fl

6. MCCarthyC.Timothy. (2005), Endoscopy for the small Animal Practitioner, 2005,2 ${ }^{\text {nd }}$ Elsevier Saunders, 60-90, 281-320 p

7. Moon JH, Kang BT, Kwon DH, Lee HC, Jeon JH, Cho KW, Jung HW, Jung DI, (2012), Esophageal and gastric endoscopic foreign body removal of 19 dogs (2009-2011), Future information Technology, Application, and Service, 179:123-128

8. ShiblyS, Karl S, Hittmair KM, Hirt RA, (2014), Acute gastroesophageal intussusception in a juvenile Australian shepherd dog: endoscopic treatment and long term follow-up, BMC Vet Res, 10:109-115

9. Tams TR, (1999), Small Animal Endoscopy, $2^{\text {nd }}$ edition, Mosby Inc., 39, 90-110 p 